rates are similar to those reported among French and US children. Recent observed increases in prevalence of autism in California and Denmark have occurred well after the introduction of the MMR vaccine and may possibly be related to heightened awareness of the diagnosis among physicians. The role of vaccines in certain neuropsychiatric illness is often controversial, but a link between MMR and autism appears to be unlikely.

\title{
ABNORMAL FRONTAL AND TEMPORAL LOBE ASYMMETRIES IN AUTISM
}

Regional cortical volume asymmetry patterns, particularly those associated with language function, in 16 boys with autism (aged 7-11 years) were compared with measures in 15 normal, age- and handedness-matched controls, in an MRI study at the Massachusetts General Hospital, Boston, and other centers. Boys with autism had significant asymmetry reversal in frontal language-related cortex: $27 \%$ larger on the right in autism and $17 \%$ larger on the left in normal controls. In addition, the posterior temporal fusiform gyrus was more left-sided in autism, whereas adjacent fusiform gyrus and temporooccipital inferior temporal gyrus were more right-sided in autism. Inferior temporal regions are involved in visual social/face processing. Abnormal structural asymmetries in language and face processing cerebral regions may relate to language and social disturbances common in autism. (Herbert MR, Harris GJ, Adrien KT, et al. Abnormal asymmetry in language association cortex in autism. Ann Neurol November 2002;52:588-596). (Respond: Dr Herbert, Center for Morphometric Analysis, Massachusetts General Hospital, CNY-149, Room 6012, Boston, MA 02114).

COMMENT. Both frontal (Broca) and temporal (Wernicke) language-related association cortex regions show a reversal of assymetry in boys with autism compared to controls, but the frontal abnormality is significantly greater. Previous SPECT and PET regional cerebral blood flow studies also show similar asymmetry reversals. MRI studies in normal adults show that the left hemisphere cortical regions are larger in right-handed, left-hemisphere language dominant subjects. In children with specific language impairment (SLI), MRI studies have shown abnormal asymmetries in language cortical measures, similar to those observed in autistic children. Language abnormalities in autism share many features of those in SLI, and these studies support a link between the two disorders.

\section{$\underline{\text { SEIZURE DISORDERS }}$}

\section{EXERCISE-INDUCED TEMPORAL LOBE EPILEPSY}

Two patients, ages 16 and 28, with left temporal lobe seizures induced by exercise are reported from Austin and Repatriation Medical Centre, Victoria, Australia. Patient 1 had seizures precipitated by physical activity from age 12 years. An aura was followed by blank stare, orobuccal automatisms, and postictal confusion. The seizures occurred within 5 to 20 minutes of commencing running and playing soccer or tennis. Other family members had seizures but none was exercise induced. Interictal EEG showed left temporal slowing, and during video EEG monitoring, two complex partial seizures were induced by stair stepping for 5 minutes. MRIs were normal, but interictal PET scan showed left temporal hypometabolism. Reduced exercise and topiramate were followed by seizure control for 7 months. Patient 2 had seizures from age 21. They began with deja vu and decreased awareness, followed by staring, and oral and bimanual automatisms. 